OPEN ACCESS

Edited by:

Ovidiu Constantin Baltatu, Anhembi Morumbi University, Brazil

Reviewed by:

Abdu Adem,

United Arab Emirates University,

United Arab Emirates Michael Huang,

The University of Sydney, Australia

*Correspondence:

Michal Mielcarek

mielcarekm/@gmail.com;

m.mielcarek@imperial.ac.uk

Specialty section:

This article was submitted to Integrative Physiology,

a section of the journal

Frontiers in Physiology

Received: 18 December 2017

Accepted: 30 April 2018

Published: 23 May 2018

Citation:

Critchley BJ, Isalan M and Mielcarek M (2018) Neuro-Cardio Mechanisms in Huntington's Disease

and Other Neurodegenerative

Disorders. Front. Physiol. 9:559.

doi: 10.3389/fphys.2018.00559

\section{Neuro-Cardio Mechanisms in Huntington's Disease and Other Neurodegenerative Disorders}

\author{
Bethan J. Critchley ${ }^{1,2}$, Mark Isalan ${ }^{1,2}$ and Michal Mielcarek ${ }^{1,2,3 *}$ \\ ${ }^{1}$ Department of Life Sciences, Imperial College London, London, United Kingdom, ${ }^{2}$ Imperial College Centre for Synthetic \\ Biology, Imperial College London, London, United Kingdom, ${ }^{3}$ Department of Epidemiology of Rare Diseases and \\ Neuroepidemiology, University of Medical Sciences, Poznań, Poland
}

Although Huntington's disease is generally considered to be a neurological disorder, there is mounting evidence that heart malfunction plays an important role in disease progression. This is perhaps not unexpected since both cardiovascular and nervous systems are strongly connected - both developmentally and subsequently in health and disease. This connection occurs through a system of central and peripheral neurons that control cardiovascular performance, while in return the cardiovascular system works as a sensor for the nervous system to react to physiological events. Hence, given their permanent interconnectivity, any pathological events occurring in one system might affect the second. In addition, some pathological signals from Huntington's disease might occur simultaneously in both the cardiovascular and nervous systems, since mutant huntingtin protein is expressed in both. Here we aim to review the source of HD-related cardiomyopathy in the light of recently published studies, and to identify similarities between HD-related cardiomyopathy and other neuro-cardio disorders.

Keywords: neuro-cardio disorders, Huntington's disease, cardiomyopathy, CNS, heart failure

\section{INTRODUCTION}

Huntington's disease (HD), reviewed in Zielonka et al. (2015), is the most common hereditary neurodegenerative disorder. It is invariably fatal and affects approximately 700,000 people worldwide (1 in 10,000). This is likely to be an underestimate of prevalence since a recent screen of healthy individuals reported that 1 in 400 might be at risk of developing HD (Kay et al., 2016). HD is caused by the expansion of a polyglutamine stretch within the huntingtin protein (HTT), which results in the formation of cytotoxic cell products. Poly-CAG DNA repeats in exon1 of the huntingtin (HTT) gene code for polyglutamines, and these repeats increase in number in diseased individuals (Walker, 2007). Healthy subjects have fewer than 35 CAG repeats, whereas HD patients range from 35 to $\sim 200$, and repeat number correlates with the age of disease onset (Walker, 2007). In rare cases $(\sim 1$ in 100$)$, the HTT gene is apparently not expanded, yet individuals present with clinical manifestations of HD. These cases are classified as HD phenocopies - for a summary see Nguyen et al. (2017).

The most widely known feature of $\mathrm{HD}$ is neurodegeneration, and this is particularly prevalent in the striatal nuclei, basal ganglia, and cerebral cortex, resulting in neurological symptoms that involve motor, cognitive, and psychiatric disturbances (Zielonka et al., 2015). As a result, patients develop a range of clinical symptoms including personality changes, motor impairment, dementia, and weight loss (Zielonka et al., 2015). In the juvenile form of HD, defined by CAG repeat length 
of 60 or above and HD diagnosis at 21 years of age or younger, additional symptoms are present, including bradykinesia, muscular hyperkinesis with stiffness, sleep disturbances, tics, moderate to severe leg pain, itching, and psychosis (Moser et al., 2017).

Although brain pathology is the best studied hallmark of HD pathology, HTT is expressed in many other tissues and organs in mammals (Strong et al., 1993). However, to date little is known about the subcellular localization of HTT in CNS and peripheral tissues. Interestingly, HTT exhibits nuclear localization in cultured neuronal cells and cytoplasmic localization in nonneuronal HEK293 cultures, which may be important for its function in different cell types (Tunah, 2012). The protein is an important component in multiple vital cellular processes such as transcription, protein trafficking, and vesicle transport ( $\mathrm{Li}$ and $\mathrm{Li}$, 2004), hence HD has been identified as a multi-system disorder (Mielcarek, 2015).

The CAG expansion within the HTT gene leads to structural changes within the mutated protein that makes mutant HTT prone to aggregation (Davies et al., 1999). Such aggregation is widespread in the brain and other peripheral tissues (Moffitt et al., 2009) but not in HD hearts (Mielcarek et al., 2014b). Regardless of whether they lead to protein aggregates, HD mutations potentially cause both loss and gain of functions in multifunctional HTT. These may manifest as an altered set of protein interactions; for example, the mutant form of HTT binds to specific histone acetylases (HATs) (Abel and Zukin, 2008) or histone deacetylases, e.g., HDAC4 (Mielcarek et al., 2013). These are global gene regulators which may have a profound effect on gene expression when perturbed, and may be vital for understanding peripheral HD pathologies.

One of the most studied peripheral pathologies in $\mathrm{HD}$ is skeletal muscle atrophy (reviewed in Zielonka et al., 2014a; Mielcarek and Isalan, 2015; Mielcarek et al., 2015a, 2017). In fact, a recent study showed that rescuing skeletal muscle degeneration alone had a profound therapeutic effect in HD model mice. This was achieved using myostatin inhibition, resulting in reduced loss of muscle mass and grip strength impairment during the expected life span of an R6/2 mouse. More importantly, it delayed onset of end-stage HD by approximately $20 \%$ of the R6/2 mouse life span (Mielcarek et al., 2014c). Since the pharmacological intervention took place specifically in skeletal muscle, i.e., peripherally to the CNS, these findings show that in principle the CNS-skeletal muscle axis can be successfully targeted on the peripheral end. This underlines the importance of peripheral pathology as a potential stand-alone therapeutic target in HD.

In this review, we aim to concentrate on the source of HD-related cardiomyopathy. We summarize pathological cardiovascular events in HD mouse models, both in the presymptomatic phase, when no behavioral abnormalities are present, and the symptomatic phase, where behavioral and molecular events are fully present. This is followed by an exploration of the cardiovascular abnormalities present in the symptomatic stage of disease in human patients. Finally, we examine similarities between HD-related cardiomyopathy and the cardiovascular component of other neurodegenerative diseases.

\section{HEART PATHOLOGICAL EVENTS IN PRE-SYMPTOMATIC HD MOUSE MODELS}

The biological function of HTT remains unknown not only in the heart, but also in other tissues including the CNS. However, proof-of-concept studies underpinning mutant HTT toxicity in the heart have been performed in various animal models. An artificial transgenic polyQ-expressing mouse model has been reported to develop heart failure, which led to premature death at eight months of age (Pattison et al., 2008). These mice expressed either a 19-glutamine control polyQ peptide (PQ19), or an 83-glutamine mutant polyQ peptide (PQ83), under regulatory control of the $\alpha$-myosin heavy chain promoter (MyHC) which drives cardiomyocyte-specific expression. The results appear to indicate an intrinsic toxic function of mutant HTT in HD hearts (Pattison et al., 2008), despite no wide-spread CNS pathology.

A similar study was performed in a Drosophila model where mutant exon-1 HTT was expressed under the control of the Hand1 (heart) promoter. This model developed an increased incidence of arrhythmias and extreme cardiac hypertrophy, accompanied by a significant decrease in contractility (Melkani et al., 2013).

Both these models clearly indicate an intrinsic toxic function of mutant HTT in HD hearts that is independent of CNS pathology. However, these experimental conditions are somewhat different from the natural pathology (where no high molecular weight aggregates are observed; Mielcarek et al., 2014b), since both models highly over-expressed either polyQ(83) peptide or mutant exon-1 HTT in cardiac tissue. On the other hand, a number of human heart failure samples of various etiologies have shown accumulation of intracellular preamyloid oligomers and higher-order assemblies (Sanbe et al., 2005). In a model of desmin-related cardiomyopathy, produced by expressing the mutant form of the cardiomyocyte-specific transgene $\alpha \mathrm{B}$-crystallin $\left(\mathrm{CryAB} \mathrm{B}^{\mathrm{R} 120 \mathrm{G}}\right.$ ), aggregates were observed within cardiomyocytes which led to altered cardiomyocyte function, perturbations in mitochondrial-sarcomere architecture, and deficits in mitochondrial function, which collectively caused apoptosis and heart failure (Maloyan et al., 2005).

In HD mouse models, several groups published data describing heart pathological events in symptomatic animals and at the end-stage of disease (Figure 1). However, there is growing evidence that some pathological events in HD hearts occur prior to CNS degeneration. For example, it has been shown that Connexin-43 in gap junctions dislocates from the end plate towards the lateral membrane in cardiomyocytes as early as 4 weeks of age in R6/2 mice, and 8 months of age in $H d h \mathrm{Q} 150$ mice, while its protein levels remained unchanged (Mielcarek et al., 2014b). This might be a first link to conduction disturbances and arrhythmogenesis in many heart diseases (Delmar and Makita, 2012). It will be vital for future 


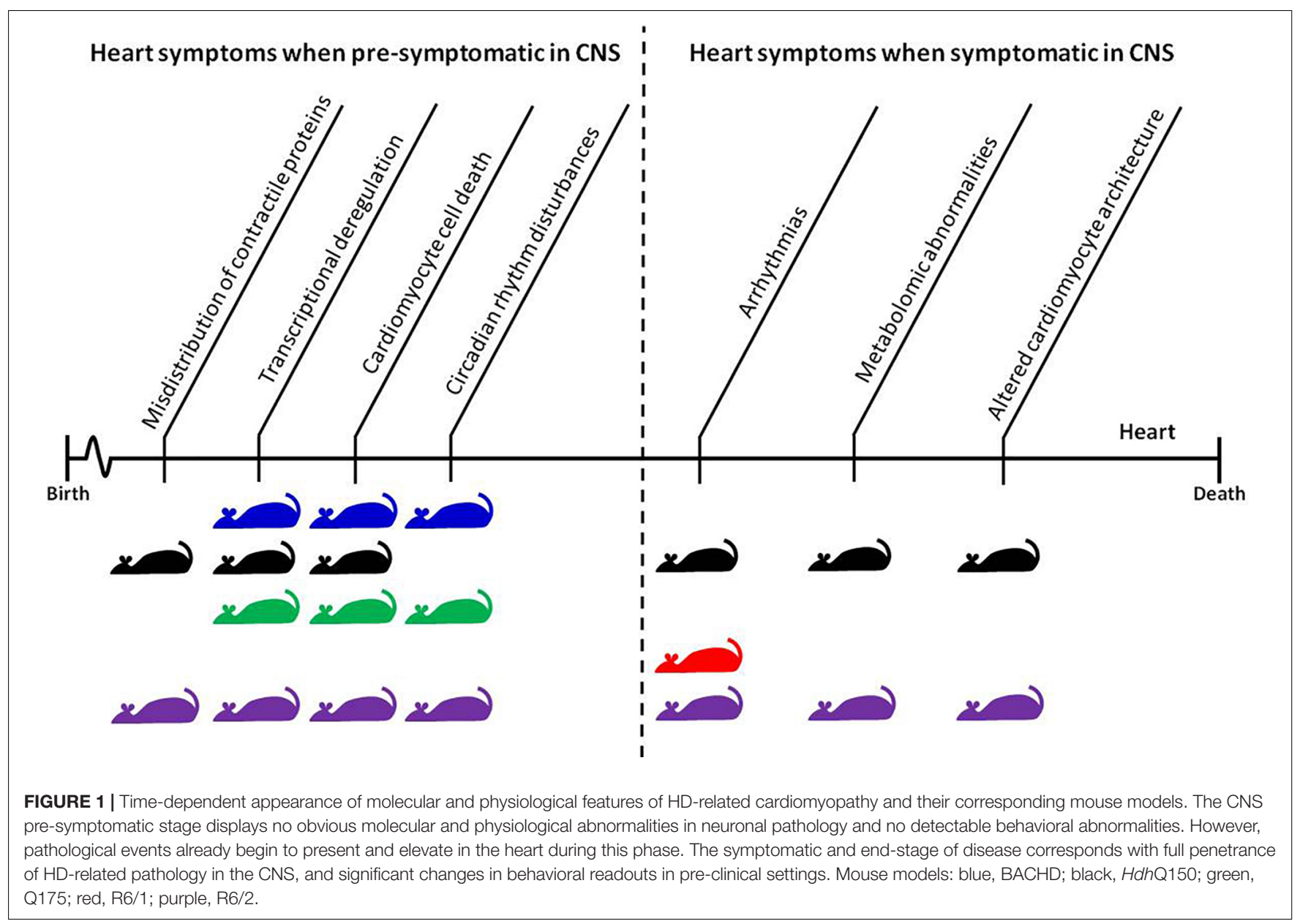

therapeutic avenues to validate whether mutant HTT also alters expression/distribution of other heart contractile proteins (Yin et al., 2015). The connexin-43 relocation was accompanied by a significant deregulation of hypertrophic markers and $B d n f$ transcripts, a significant degree of fibrosis, and an increased level of apoptotic nuclei in pre-symptomatic mouse models (Mielcarek et al., 2014b). In the BACHD mouse model, the first structural and functional differences (i.e., end systolic dimension) have been detected in pre-symptomatic animals as early as 3 months of age, and continued throughout their life span. At this early stage, HD mice displayed profound transcriptional changes related to key biological process including apoptosis, gene expression, proliferation, and proteolysis/ubiquitination (Schroeder et al., 2016). In addition, the young mice showed evidence of an upregulated pro-inflammatory immune response, particularly with respect to IL-6 levels, which could contribute to the observed cardiopathological changes (Schroeder et al., 2016).

Recently, another pathological component of the cardiovascular system has been identified pre-symptomatically in the R6/2 mouse model. These mice showed a significant enhancement of endothelial-dependent dilation and impairment of $\alpha 1$ adrenergic vasoconstrictor responses. Consequently, the mice failed to recruit NO-dependent pathways and displayed increased endothelial-mediated relaxation. In addition, symptomatic R6/2 mice had significantly altered endothelial function in peripheral resistance arteries (Kane et al., 2016). Moreover, $\mu \mathrm{MRA}$ (three-dimensional microscopic magnetic resonance angiography) revealed an increase in vessel volume fraction and cerebral blood volume in the brains of pre-symptomatic R6/2 mice. Collagen IV immunostaining revealed an enrichment in vessel density, but no increase in vessel size, within the microvasculature of the mouse HD brain, which worsened with disease progression. Most importantly, vessel densities in the cortex, caudate/putamen, and substantia nigra were higher in early symptomatic HD patients compared to non-HD human subjects (Lin et al., 2013). Taken together, these data suggest a link between $\mathrm{HD}$ and changes in vasculature.

In addition, symptomatic Q175 knock-in mice demonstrate disturbances to circadian and diurnal rhythms, including blunted daily cycling in heart rate and heart rate variability as well as autonomic dysfunction (demonstrated by lack of core body temperature regulation and poor performance in the baroreceptor challenge) (Cutler et al., 2017). Similar circadian disturbances have been seen in BACHD and R6/2 mice (Kudo et al., 2011). In conclusion, there is mounting evidence that circadian rhythms are impaired both in HD mouse models and in $\mathrm{HD}$ patients; this may have profound consequences 
for heart malfunction with the source being the CNS axis end.

\section{HEART PATHOLOGICAL EVENTS IN SYMPTOMATIC HD MOUSE MODELS}

Recently, several studies have examined heart malfunction in the symptomatic and late-end stage of HD using different HD mouse models which are characterized by time-dependent disease progression (see Figure $\mathbf{1}$ for an overview). Early pathological events in the heart such as connexin- 43 dislocation, transcriptional deregulation, and apoptotic cell death were elevated throughout the life span of $\mathrm{R} 6 / 2$ and $H d h \mathrm{Q} 150$ mouse models (Mielcarek et al., 2014b). This was accompanied by an increased level of fibrotic deposits in both $\mathrm{R} 6 / 2$ and HdhQ150 (Mielcarek et al., 2014b). In Q175 knock-in mice, pockets of fibrosis were observed in the interventricular septum, and the size of cardiomyocytes was reduced, both of which exhibited age-dependent progression (Cutler et al., 2017). Similarly, symptomatic BACHD mice develop cardiac fibrosis and ultimately apoptosis (Schroeder et al., 2016). In fact, cardiac Fas-dependent and mitochondria-dependent apoptotic pathways have been identified to be activated in the hearts of symptomatic R6/2 mice. This was accompanied by increased levels of crucial components of both Fas-dependent apoptosis (TNFalpha, TNFR1, Fas ligand, Fas death receptors, FADD, activated caspase-8, and activated caspase-3) and mitochondria-dependent apoptosis (Bax, Bax-to- Bcl-2 ratio, cytosolic cytochrome c, activated caspase-9, and activated caspase-3) (Wu et al., 2015). Furthermore, during disease progression, the number of transcripts with altered expression increased significantly in R6/2 and HdhQ150 mouse models (Mielcarek et al., 2014b) as well in BACHD mice (Schroeder et al., 2016). Therefore cardiac molecular changes are observed across many HD mouse models.

In symptomatic HD mouse models, pronounced functional changes were visualized by cardiac MRI, revealing a number of abnormalities. These may contribute to dilated cardiomyopathy (DCM) in the R6/2 mouse model (Wood et al., 2012) and HdhQ150 mice (Mielcarek et al., 2014b). At the physiological level, HD hearts also displayed a contractile dysfunction based on ECG measurements in two HD mouse models (R6/2 and $H d h \mathrm{Q} 150)$ (Mielcarek et al., 2014b). This was supported by molecular pathological events including altered architecture of ganglionic plexuses (observed using tyrosine hydroxylase staining) and lower levels of $B d n f$ transcripts in $\mathrm{HD}$ murine hearts (Mielcarek et al., 2014b). The R6/1 model, which had unstable RR intervals that were reversed following atropine treatment, shows similar molecular pathological events, suggesting parasympathetic nervous activation. The mice developed brady- and tachyarrhythmias, including paroxysmal atrial fibrillation and cardiac sudden death (Kiriazis et al., 2012).

Since myocardial contraction depends strongly on mitochondrial energy supply, it is likely that HD murine hearts could display alterations in cardiac energy equilibrium.
Consequently, an investigation of both $\mathrm{R} 6 / 2$ and $H d h \mathrm{Q} 150$ mouse models showed that they have reduced glucose usage and a significant deregulation of genes involved in de novo purine biosynthesis, conversion of adenine nucleotides, and adenosine metabolism (despite AMP-activated protein kinase hyperphosphorylation) (Toczek et al., 2016b). At the molecular level, increased levels of nucleotide catabolites such as inosine, hypoxanthine, xanthine, and uric acid and decreased levels of adenosine have been observed in murine and human HD serum. These metabolites represent the first identified biomarkers related to striated muscle dysfunction in $\mathrm{HD}$, in both pre-clinical and clinical settings (Toczek et al., 2016b). High-performance liquid chromatography assays demonstrate that enzymatic activity is also affected; AMPD and e $5^{\prime} \mathrm{NT}$ exhibit decreased activity whereas ADA activity levels are increased, suggesting that mutant HTT could be disrupting cardiac nucleotide metabolism on a transcriptional level (Toczek et al., 2016a). Importantly, mutant HTT interacts with global gene regulators, such as HDACs, and this is potentially important for the gene expression changes observed in HD, as well as other types of cardiomyopathies (Mielcarek et al., 2013, 2015b; Piotrowska et al., 2017).

Rodent heart disease models also exhibit alterations in cardiac energy equilibrium, with rat aortic constriction models showing reduced glucose metabolism similar to R6/2 and HdHQ150 HD models (Doenst et al., 2013). Furthermore, models of myocardial infarction, hypertension, renal failure, and aortic constriction provide evidence for mitochondrial dysfunction or deregulated mitochondrial gene expression (Doenst et al., 2013). Overall, these similarities between rodent models of $\mathrm{HD}$ and heart failure do suggest that HD-related pathology could contribute to heart failure due to the disruption of the cardiac energy balance.

\section{HD RELATED CARDIOMYOPATHY IN THE CLINIC}

Since Huntington's disease has been described in the literature as an exclusively neurological disorder, there are a very limited number of studies evaluating heart function in HD patients. This is despite previously published epidemiological data that clearly identified heart failure as the second most common cause of death in HD (reviewed in Zielonka et al., 2014b). Only very recently, a clinical study by Stephen et al. (2015) on a large cohort of early symptomatic patients revealed significant contractile heart dysfunction. The study was performed using standard 12-lead electrocardiograms (ECGs) and found that abnormal ECGs were typical for $25.3 \%$ of early symptomatic patients. Abnormalities were manifested in several ways, including bradycardia and prolonged QTc interval and/or intra-ventricular conduction, likely leading to arrhythmia and aggravated cardiac failure. An additional study on $41 \mathrm{HD}$ subjects found significantly greater arterial stiffness (as a result of diminished vascular smooth muscle contractility due to autonomic dysfunction) in presymptomatic and early-stage $\mathrm{HD}$ as well as increased intima media thickness in mid and late stages (Kobal et al., 2017). These findings support those from multiple studies of different HD 
mouse models, which also revealed contractile heart dysfunction at various stages of disease progression (Kiriazis et al., 2012; Mielcarek et al., 2014b; Schroeder et al., 2016).

In addition, several clinical reports performed on small cohorts of early or symptomatic patients revealed that HD patients have enhanced cardiovagal activity (Andrich et al., 2002), reduced heart rate (Melik et al., 2012), and 10.6\% lower diastolic pressure (Kobal et al., 2010). Unfortunately, to date there is no molecular data available that may underpin the mechanism of heart failure in human HD. The only study published so far identified a set of nucleotide catabolites in human HD sera which are associated with failing HD hearts (Toczek et al., 2016b).

\section{SHARED PATHOLOGICAL EVENTS IN HD-RELATED CARDIOMYOPATHY AND OTHER CARDIO-NEURO DISEASES}

It is well known that the canonical approach to studying any neurodegenerative disease focuses exclusively on neuronal defects caused by inherited or non-inherited factors. This is against a school of thought suggesting a permanent interconnectivity between the cardiovascular and nervous systems. Since both systems are directly linked, any pathological insult causing malfunction in one of them may have an indirect impact on the other. Multiple neuro-cardio diseases demonstrate their interconnectivity - Huntington's disease serves as a good example because several of the cardiac dysfunctions which present over the course of disease progression are controlled by brain regions affected during HD pathology (Figure 2). As such, an approach towards finding novel cardiac therapeutic avenues might at least delay HD symptoms.

There are numerous hereditary and non-hereditary neurodegenerative diseases, in which cardiovascular malfunction has been identified as a leading element in disease progression, often manifesting as arrhythmias, cardiomyopathy, or autonomic dysfunction. Cardiomyopathy is the most common cause of death and affects $66 \%$ of patients with FA (Friedreich's ataxia), an autosomal recessive neuro and cardio-degenerative disorder that is caused by deficient expression of the nuclear-encoded mitochondrial protein frataxin (Lane et al., 2013). Patients often present with left ventricular hypertrophy, which can develop into more fatal dilated cardiomyopathy (Lane et al., 2013). Moreover, FA cardiomyocytes are significantly larger than control cells and are surrounded by fibrotic endomysium (Koeppen et al., 2015). There are numerous mouse models of FA that develop heart cardiomyopathy reflecting clinical endpoints (Payne et al., 2011). Similarly, large numbers of fibrotic deposits and cardiomyocyte disarray has been identified in pre-clinical models of HD (Mielcarek et al., 2014b; Schroeder et al., 2016).

Neurodegenerative disorders like Parkinson's disease (PD) (Dauer and Przedborski, 2003; Amino et al., 2005) are often accompanied by fatigue, dysrhythmias, and dysregulation in the electrical activity, i.e., prolongation in the corrected QTc interval or reduced heart rate variability (reviewed in Joers and Emborg, 2014). Interestingly, although originally described as a movement disorder linked to the loss of dopaminergic neurons, PD has recently been classified as a multisystem disorder affecting both central and peripheral nervous systems (Dauer and Przedborski, 2003; Amino et al., 2005). Arrhythmias have also been observed in bulbar spinal muscular atrophy (BSMA), which could be caused by myocardial accumulation of mutant androgen receptor protein, analogous to Lewy body formation in PD neurons (Araki et al., 2014). There is also evidence to suggest that Lewy bodies occur in epicardial nerve fascicles prior to CNS degeneration (Navarro-Otano et al., 2013), and similar findings have been reported in HD pre-clinical and clinical settings (Mielcarek et al., 2014b; Stephen et al., 2015).

Familial Alzheimer's disease (AD) is predominantly caused by presenilin 1 (PSEN1) or presenilin 2 (PSEN2) mutations (Bird, 2005). The presenilins are also expressed in the heart and have been found to be critical to cardiac development (Donoviel et al., 1999; Nakajima et al., 2004). PSEN1 mutation has been associated with complete penetrance and progressive disease that necessitates cardiac transplantation or results in death (Li et al., 2006). By contrast, PSEN2 mutation results in partial penetrance, milder disease, and a more favorable prognosis. Nonetheless, it is striking that both mutations are associated with a higher risk of dilated cardiomyopathy and heart failure in $\mathrm{AD}$ patients (Li et al., 2006).

$\mathrm{AD}$ also has a cardiovascular defect (similar to HD) associated with microvascular dysfunction and/or neurovascular disintegration. Microvascular deficits diminish CBF and, subsequently, the brain's supply of oxygen, energy substrates, and nutrients (reviewed in Guo and Lo, 2009; Zlokovic, 2011).

In terms of signaling, the APP/PS1 AD model shows reduced responsiveness to adrenergic agonists, although $\beta_{1^{-}}$ adrenergic receptor expression is apparently unchanged in APP/PS1 hearts (Turdi et al., 2009). This is similar to the lack of responsiveness to $\beta_{1}$-adrenergic receptor stimulation which has been observed in symptomatic $\mathrm{HD}$ R6/2 mice when chronically treated with isoproterenol, a potent betaadrenoreceptor agonist (Mielcarek et al., 2014a). Moreover, representative heart sections in the primary literature show BACHD symptomatic mouse hearts, treated with isoproterenol, as having extremely high levels of fibrosis, much more than would be expected with this drug treatment (Schroeder et al., 2016). In this case, the heart muscle appears to be dilated as opposed to hypertrophic. Pre-symptomatic BACHD mice respond adaptively to isoproterenol treatment, suggesting that response failure to adrenergic signaling only manifests once behavioral and molecular changes have emerged (Schroeder et al., 2016).

Finally, Timothy syndrome (TS) is a multi-system disorder which affects both the nervous and cardiac systems and is caused by a missense point mutation in the CACNA1C gene (Dixon et al., 2012). Similar to HD, patients suffer from a prolonged QT interval and structural heart defects in addition to neurodevelopmental delay, seizures, and autism-spectrum disorder symptoms (Splawski et al., 2004). Other neuro-cardio diseases may exhibit partially penetrant symptom crossover with HD cardiac pathology. For instance, around $50 \%$ of patients with Amyotrophic lateral sclerosis (ALS) may be affected by chronic 


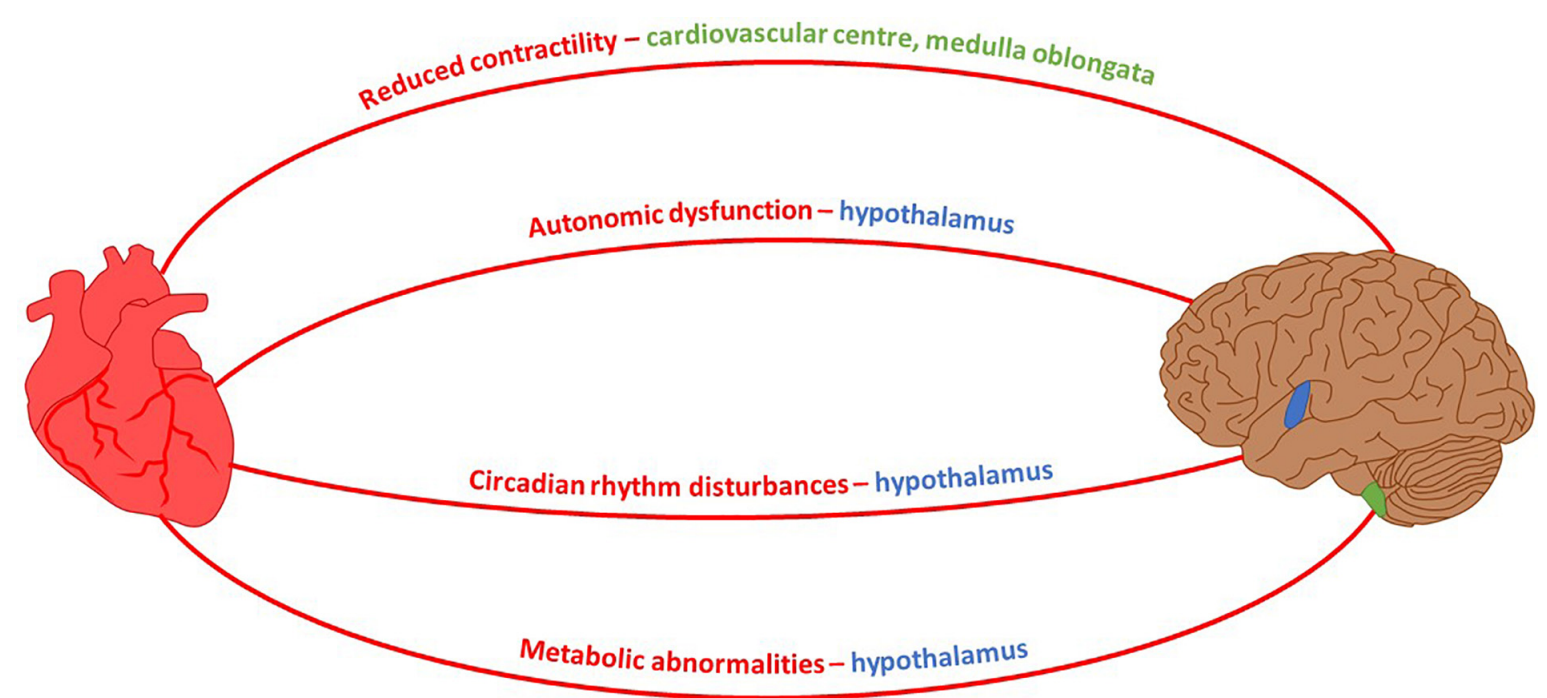

FIGURE 2 | Neuro-Cardio connections between HD-related cardiomyopathy and brain regions affected by HD. Mouse model HD symptoms and their associated brain control region which exhibits degeneration during the course of HD pathology. Shaded regions of the brain: blue, hypothalamus; green, medulla oblongata (Petersen and Gabery, 2012; Rub et al., 2014).

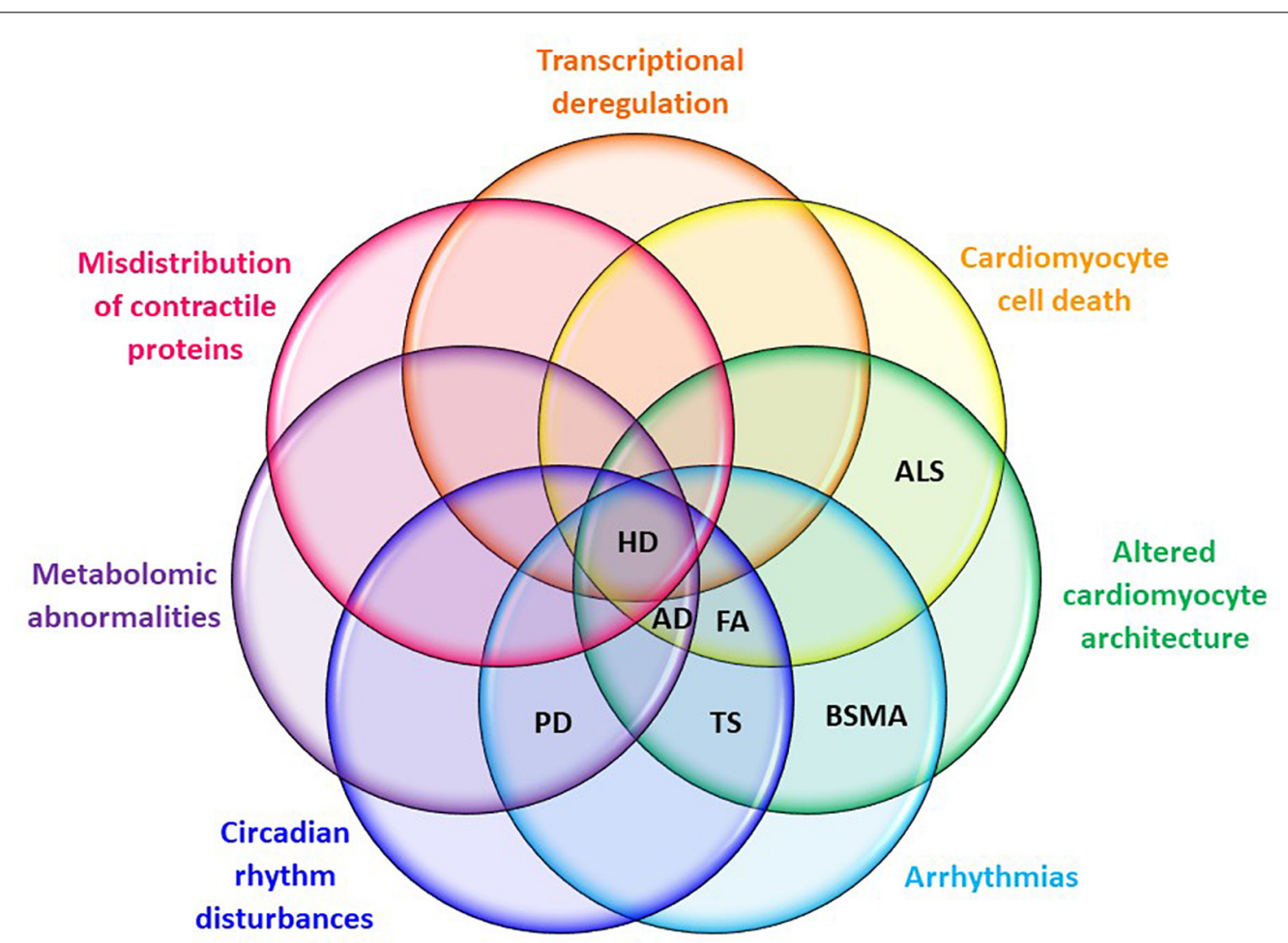

FIGURE 3 | Cardiac symptom overlaps between HD and other neuro-cardio diseases. Symptoms listed are those evident in mouse models of HD at CNS pre-symptomatic or symptomatic stages. AD, Alzheimer's disease; ALS, amyotrophic lateral sclerosis; BSMA, bulbar spinal muscular atrophy; FA, Friedreich's ataxia; HD, Huntington's disease; PD, Parkinson's disease; TS, Timothy syndrome.

cardiac sympathetic hyperactivity, which is linked to sudden cardiac death and stress-induced cardiomyopathy - similar to the cardiomyopathy seen in pre-clinical and clinical HD (Tanaka et al., 2013). Additionally, ALS patients have elevated resting blood pressure and, in contrast to the diminished response to $\beta_{1}$-adrenergic receptor stimulation in $\mathrm{HD}$ and $\mathrm{AD}$ patients, exhibit increased response to alpha-adrenergic stimulation via administration of phentolamine (Tanaka et al., 2013). 


\section{CONCLUSION}

Huntington's disease shares many pathological features with other neurodegenerative disorders, including peripheral organ malfunction in the heart (see Figure $\mathbf{3}$ for a summary of shared cardiopathological symptoms). Recent studies provide new and strong evidence that HD-related cardiomyopathy plays a detrimental role in disease progression. However, urgent studies are now needed to fully understand not only the pathological consequences of HD-related cardiomyopathy, but also to underpin its molecular source using genetic approaches. This new understanding will be beneficial in order to fully ameliorate all pathological features of HD, allowing the development of new therapeutic strategies.

\section{REFERENCES}

Abel, T., and Zukin, R. S. (2008). Epigenetic targets of HDAC inhibition in neurodegenerative and psychiatric disorders. Curr. Opin. Pharmacol. 8, 57-64. doi: 10.1016/j.coph.2007.12.002

Amino, T., Orimo, S., Itoh, Y., Takahashi, A., Uchihara, T., and Mizusawa, H. (2005). Profound cardiac sympathetic denervation occurs in Parkinson disease. Brain Pathol. 15, 29-34. doi: 10.1111/j.1750-3639.2005.tb00097.x

Andrich, J., Schmitz, T., Saft, C., Postert, T., Kraus, P., Epplen, J. T., et al. (2002). Autonomic nervous system function in Huntington's disease. J. Neurol. Neurosurg. Psychiatry 72, 726-731. doi: 10.1136/jnnp.72.6.726

Araki, A., Katsuno, M., Suzuki, K., Banno, H., Suga, N., Hashizume, A., et al. (2014). Brugada syndrome in spinal and bulbar muscular atrophy. Neurology 82, 1813-1821. doi: 10.1212/WNL.0000000000000434

Bird, T. D. (2005). Genetic factors in Alzheimer's disease. N. Engl. J. Med. 352, 862-864. doi: 10.1056/NEJMp058027

Cutler, T. S., Park, S., Loh, D. H., Jordan, M. C., Yokota, T., and Roos, K. P. (2017). Neurocardiovascular deficits in the Q175 mouse model of Huntington's disease. Physiol. Rep. 5:e13289. doi: 10.14814/phy2.13289

Dauer, W., and Przedborski, S. (2003). Parkinson's disease: mechanisms and models. Neuron 39, 889-909. doi: 10.1016/S0896-6273(03)00568-3

Davies, S. W., Sathasivam, K., Hobbs, C., Doherty, P., Mangiarini, L., Scherzinger, E., et al. (1999). Detection of polyglutamine aggregation in mouse models. Methods Enzymol. 309, 687-701. doi: 10.1016/S0076-6879(99)09045-X

Delmar, M., and Makita, N. (2012). Cardiac connexins, mutations and arrhythmias. Curr. Opin. Cardiol. 27, 236-241. doi: 10.1097/HCO.0b013e328352220e

Dixon, R. E., Cheng, E. P., Mercado, J. L., and Santana, L. F. (2012). L-type $\mathrm{Ca} 2+$ channel function during Timothy syndrome. Trends Cardiovasc. Med. 22, 72-76. doi: 10.1016/j.tcm.2012.06.015

Doenst, T., Nguyen, T. D., and Abel, E. D. (2013). Cardiac metabolism in heart failure: implications beyond Atp production. Circ. Res. 113, 709-724. doi: 10. 1161/CIRCRESAHA.113.300376

Donoviel, D. B., Hadjantonakis, A. K., Ikeda, M., Zheng, H., Hyslop, P. S., and Bernstein, A. (1999). Mice lacking both presenilin genes exhibit early embryonic patterning defects. Genes Dev. 13, 2801-2810. doi: 10.1101/gad.13.21.2801

Guo, S., and Lo, E. H. (2009). Dysfunctional cell-cell signaling in the neurovascular unit as a paradigm for central nervous system disease. Stroke 40, S4-S7. doi: 10.1161/STROKEAHA.108.534388

Joers, V., and Emborg, M. E. (2014). Modeling and imaging cardiac sympathetic neurodegeneration in Parkinson's disease. Am. J. Nucleic Med. Mol. Imaging 4, 125-159.

Kane, A. D., Niu, Y., Herrera, E. A., Morton, A. J., and Giussani, D. A. (2016). Impaired nitric oxide mediated vasodilation in the peripheral circulation in the R6/2 mouse model of Huntington's disease. Sci. Rep. 6:25979. doi: 10.1038/ srep 25979

Kay, C., Collins, J. A., Miedzybrodzka, Z., Madore, S. J., Gordon, E. S., Gerry, N., et al. (2016). Huntington disease reduced penetrance alleles occur at high frequency in the general population. Neurology 87, 282-288. doi: 10.1212/WNL. 0000000000002858

\section{AUTHOR CONTRIBUTIONS}

All authors listed have made a substantial, direct, and intellectual contribution to the work, and approved it for publication.

\section{FUNDING}

This work was supported by the European Research Council grant H2020 - ERC-2014-PoC 641232 - Fingers4Cure; and an Imperial/ICR/NIHR BRC/NHS Confidence in Concept (iCiC) grant. These funders had no role in study design, data collection and analysis, decision to publish or preparation of the manuscript. MI was funded by Wellcome Trust UK New Investigator Award No. WT102944.

Kiriazis, H., Jennings, N. L., Davern, P., Lambert, G., Su, Y., Pang, T., et al. (2012). Neurocardiac dysregulation and neurogenic arrhythmias in a transgenic mouse model of Huntington's disease. J. Physiol. 590, 5845-5860. doi: 10.1113/jphysiol. 2012.238113

Kobal, J., Cankar, K., Pretnar, J., Zaletel, M., Kobal, L., Teran, N., et al. (2017). Functional impairment of precerebral arteries in Huntington disease. J. Neurol. Sci. 372, 363-368. doi: 10.1016/j.jns.2016.10.033

Kobal, J., Melik, Z., Cankar, K., Bajrovic, F. F., Meglic, B., Peterlin, B., et al. (2010). Autonomic dysfunction in presymptomatic and early symptomatic Huntington's disease. Acta Neurol. Scand. 121, 392-399. doi: 10.1111/j.16000404.2009.01251.x

Koeppen, A. H., Ramirez, R. L., Becker, A. B., Bjork, S. T., Levi, S., Santambrogio, P., et al. (2015). The pathogenesis of cardiomyopathy in Friedreich ataxia. PLoS One 10:e0116396. doi: 10.1371/journal.pone.0116396

Kudo, T., Schroeder, A., Loh, D. H., Kuljis, D., Jordan, M. C., Roos, K. P., et al. (2011). Dysfunctions in circadian behavior and physiology in mouse models of Huntington's disease. Exp. Neurol. 228, 80-90. doi: 10.1016/j.expneurol.2010. 12.011

Lane, D. J., Huang, M. L., Ting, S., Sivagurunathan, S., and Richardson, D. R. (2013). Biochemistry of cardiomyopathy in the mitochondrial disease Friedreich's ataxia. Biochem. J. 453, 321-336. doi: 10.1042/BJ20130079

Li, D., Parks, S. B., Kushner, J. D., Nauman, D., Burgess, D., Ludwigsen, S., et al. (2006). Mutations of presenilin genes in dilated cardiomyopathy and heart failure. Am. J. Hum. Genet. 79, 1030-1039. doi: 10.1086/509900

Li, S. H., and Li, X. J. (2004). Huntingtin-protein interactions and the pathogenesis of Huntington's disease. Trends Genet. 20, 146-154. doi: 10.1016/j.tig.2004.01.008

Lin, C. Y., Hsu, Y. H., Lin, M. H., Yang, T. H., Chen, H. M., Chen, Y. C., et al. (2013). Neurovascular abnormalities in humans and mice with Huntington's disease. Exp. Neurol. 250, 20-30. doi: 10.1016/j.expneurol.2013.08.019

Maloyan, A., Sanbe, A., Osinska, H., Westfall, M., Robinson, D., Imahashi, K., et al. (2005). Mitochondrial dysfunction and apoptosis underlie the pathogenic process in alpha-B-crystallin desmin-related cardiomyopathy. Circulation 112, 3451-3461. doi: 10.1161/CIRCULATIONAHA.105.572552

Melik, Z., Kobal, J., Cankar, K., and Strucl, M. (2012). Microcirculation response to local cooling in patients with Huntington's disease. J. Neurol. 259, 921-928. doi: 10.1007/s00415-011-6279-3

Melkani, G. C., Trujillo, A. S., Ramos, R., Bodmer, R., Bernstein, S. I., and Ocorr, K. (2013). Huntington's disease induced cardiac amyloidosis is reversed by modulating protein folding and oxidative stress pathways in the Drosophila heart. PLoS Genet. 9:e1004024. doi: 10.1371/journal.pgen.1004024

Mielcarek, M. (2015). Huntington's disease is a multi-system disorder. Rare Dis. 3:e1058464. doi: 10.1080/21675511.2015.1058464

Mielcarek, M., Bondulich, M. K., Inuabasi, L., Franklin, S. A., Muller, T., and Bates, G. P. (2014a). The Huntington's disease-related cardiomyopathy prevents a hypertrophic response in the R6/2 mouse model. PLoS One 9:e108961. doi: 10.1371/journal.pone.0108961

Mielcarek, M., Inuabasi, L., Bondulich, M. K., Muller, T., Osborne, G. F., Franklin, S. A., et al. (2014b). Dysfunction of the CNS-heart axis in mouse models 
of Huntington's disease. PLoS Genet. 10:e1004550. doi: 10.1371/journal.pgen. 1004550

Mielcarek, M., and Isalan, M. (2015). A shared mechanism of muscle wasting in cancer and Huntington's disease. Clin. Transl. Med. 4:34. doi: 10.1186/s40169015-0076-z

Mielcarek, M., Landles, C., Weiss, A., Bradaia, A., Seredenina, T., Inuabasi, L., et al. (2013). HDAC4 reduction: a novel therapeutic strategy to target cytoplasmic huntingtin and ameliorate neurodegeneration. PLoS Biol. 11:e1001717. doi: 10.1371/journal.pbio.1001717

Mielcarek, M., Rattray, I., Osborne, G. F., Jolinon, N., Dick, J. R. T., Bondulich, M. K., et al. (2014c). Myostatin inhibition as a novel approach to targeting muscle pathology in HD. J. Neurol. Neurosurg. Psychiatry 85:A97. doi: 10.1136/ jnnp-2014-309032.281

Mielcarek, M., Smolenski, R. T., and Isalan, M. (2017). Transcriptional signature of an altered purine metabolism in the skeletal muscle of a Huntington's disease mouse model. Front. Physiol. 8:127. doi: 10.3389/fphys.2017.00127

Mielcarek, M., Toczek, M., Smeets, C. J., Franklin, S. A., Bondulich, M. K., Jolinon, N., et al. (2015a). Hdac4-myogenin axis as an important marker of Hdrelated skeletal muscle atrophy. PLoS Genet. 11:e1005021. doi: 10.1371/journal. pgen. 1005021

Mielcarek, M., Zielonka, D., Carnemolla, A., Marcinkowski, J. T., and Guidez, F. (2015b). HDAC4 as a potential therapeutic target in neurodegenerative diseases: a summary of recent achievements. Front. Cell Neurosci. 9:42. doi: 10.3389/ fncel.2015.00042

Moffitt, H., Mcphail, G. D., Woodman, B., Hobbs, C., and Bates, G. P. (2009). Formation of polyglutamine inclusions in a wide range of non-CNS tissues in the HdhQ150 knock-in mouse model of Huntington's disease. PLoS One 4:e8025. doi: 10.1371/journal.pone.0008025

Moser, A. D., Epping, E., Espe-Pfeifer, P., Martin, E., Zhorne, L., Mathews, K., et al. (2017). A survey-based study identifies common but unrecognized symptoms in a large series of juvenile Huntington's disease. Neurodegener. Dis. Manag. 7, 307-315. doi: 10.2217/nmt-2017-0019

Nakajima, M., Moriizumi, E., Koseki, H., and Shirasawa, T. (2004). Presenilin 1 is essential for cardiac morphogenesis. Dev. Dyn. 230, 795-799. doi: 10.1002/dvdy. 20098

Navarro-Otano, J., Gelpi, E., Mestres, C. A., Quintana, E., Rauek, S., Ribalta, T., et al. (2013). Alpha-synuclein aggregates in epicardial fat tissue in living subjects without parkinsonism. Parkinsonism Relat. Disord. 19, 27-31. doi: 10.1016/j. parkreldis.2012.07.005

Nguyen, P. T., Meeks, D., and Liotiri, D. (2017). Anaesthesia and orphan disease: airway and anaesthetic management in Huntington's disease. BMJ Case Rep. 2017:bcr-2017-221354. doi: 10.1136/bcr-2017-221354

Pattison, J. S., Sanbe, A., Maloyan, A., Osinska, H., Klevitsky, R., and Robbins, J. (2008). Cardiomyocyte expression of a polyglutamine preamyloid oligomer causes heart failure. Circulation 117, 2743-2751. doi: 10.1161/ CIRCULATIONAHA.107.750232

Payne, R. M., Pride, P. M., and Babbey, C. M. (2011). Cardiomyopathy of Friedreich's ataxia: use of mouse models to understand human disease and guide therapeutic development. Pediatr. Cardiol. 32, 366-378. doi: 10.1007/s00246011-9943-6

Petersen, A., and Gabery, S. (2012). Hypothalamic and limbic system changes in Huntington's disease. J. Huntingtons Dis. 1, 5-16. doi: 10.3233/JHD-2012120006

Piotrowska, I., Isalan, M., and Mielcarek, M. (2017). Early transcriptional alteration of histone deacetylases in a murine model of doxorubicininduced cardiomyopathy. PLoS One 12:e0180571. doi: 10.1371/journal.pone. 0180571

Rub, U., Hentschel, M., Stratmann, K., Brunt, E., Heinsen, H., Seidel, K., et al. (2014). Huntington's disease (Hd): degeneration of select nuclei, widespread occurrence of neuronal nuclear and axonal inclusions in the brainstem. Brain Pathol. 24, 247-260. doi: 10.1111/bpa.12115

Sanbe, A., Osinska, H., Villa, C., Gulick, J., Klevitsky, R., Glabe, C. G., et al. (2005). Reversal of amyloid-induced heart disease in desmin-related cardiomyopathy. Proc. Natl. Acad. Sci. U.S.A. 102, 13592-13597. doi: 10.1073/pnas.0503324102

Schroeder, A. M., Wang, H. B., Park, S., Jordan, M. C., Gao, F., Coppola, G., et al. (2016). Cardiac dysfunction in the bachd mouse model of Huntington's Disease. PLoS One 11:e0147269. doi: 10.1371/journal.pone.0147269
Splawski, I., Timothy, K. W., Sharpe, L. M., Decher, N., Kumar, P., Bloise, R., et al. (2004). Ca(V)1.2 calcium channel dysfunction causes a multisystem disorder including arrhythmia and autism. Cell 119, 19-31. doi: 10.1016/j.cell.2004.09.011

Stephen, C., Hersch, S., and Rosas, H. (2015). Huntington's disease and the heart: electrocardiogram abnormalities suggest cardiac involvement. Neurology 84, P5.294.

Strong, T. V., Tagle, D. A., Valdes, J. M., Elmer, L. W., Boehm, K., Swaroop, M., et al. (1993). Widespread expression of the human and rat Huntington's disease gene in brain and nonneural tissues. Nat. Genet. 5, 259-265. doi: 10.1038/ng1193-259

Tanaka, Y., Yamada, M., Koumura, A., Sakurai, T., Hayashi, Y., Kimura, A., et al. (2013). Cardiac sympathetic function in the patients with amyotrophic lateral sclerosis: analysis using cardiac [123I] Mibg scintigraphy. J. Neurol. 260, 2380-2386. doi: 10.1007/s00415-013-7005-0

Toczek, M., Kutryb-Zajac, B., Zukowska, P., Slominska, E., Isalan, M., Mielcarek, M., et al. (2016a). Changes in cardiac nucleotide metabolism in Huntington's disease. Nucleosides Nucleotides Nucleic Acids 35, 707-712. doi: 10.1080/15257770.2016.1154969

Toczek, M., Zielonka, D., Zukowska, P., Marcinkowski, J. T., Slominska, E., Isalan, M., et al. (2016b). An impaired metabolism of nucleotides underpins a novel mechanism of cardiac remodeling leading to Huntington's disease related cardiomyopathy. Biochim. Biophys. Acta 1862, 2147-2157. doi: 10.1016/ j.bbadis.2016.08.019

Tunah, N. E. (2012). Modeling Huntington's Disease: in vivo, in vitro, in silico - "Huntington's Disease - Core Concepts and Current Advances". Rijeka: Intechopen.

Turdi, S., Guo, R., Huff, A. F., Wolf, E. M., Culver, B., and Ren, J. (2009). Cardiomyocyte contractile dysfunction in the APPswe/PS1dE9 mouse model of Alzheimer's disease. PLoS One 4:e6033. doi: 10.1371/journal.pone.000 6033

Walker, F. O. (2007). Huntington's disease. Lancet 369, 218-228. doi: 10.1016/ S0140-6736(07)60111-1

Wood, N. I., Sawiak, S. J., Buonincontri, G., Niu, Y., Kane, A. D., Carpenter, T. A., et al. (2012). Direct evidence of progressive cardiac dysfunction in a transgenic mouse model of Huntington's disease. J. Huntingtons Dis. 1, 57-64. doi: 10.3233/JHD-2012-120004

Wu, B. T., Chiang, M. C., Tasi, C. Y., Kuo, C. H., Shyu, W. C., Kao, C. L., et al. (2015). Cardiac Fas-Dependent and mitochondria-dependent apoptotic pathways in a transgenic mouse model of Huntington's Disease. Cardiovasc. Toxicol. 16, 111-121. doi: 10.1007/s12012-015-9318-y

Yin, Z., Ren, J., and Guo, W. (2015). Sarcomeric protein isoform transitions in cardiac muscle: a journey to heart failure. Biochim. Biophys. Acta 1852, 47-52. doi: 10.1016/j.bbadis.2014.11.003

Zielonka, D., Mielcarek, M., and Landwehrmeyer, G. B. (2015). Update on Huntington's disease: advances in care and emerging therapeutic options. Parkinsonism Relat. Disord. 21, 169-178. doi: 10.1016/j.parkreldis.2014. 12.013

Zielonka, D., Piotrowska, I., Marcinkowski, J. T., and Mielcarek, M. (2014a). Skeletal muscle pathology in Huntington's disease. Front. Physiol. 5:380. doi: 10.3389/fphys.2014.00380

Zielonka, D., Piotrowska, I., and Mielcarek, M. (2014b). Cardiac dysfunction in Huntington's Disease. Exp. Clin. Cardiol. 20, 2547-2554.

Zlokovic, B. V. (2011). Neurovascular pathways to neurodegeneration in Alzheimer's disease and other disorders. Nat. Rev. Neurosci. 12, 723-738. doi: $10.1038 / \mathrm{nrn} 3114$

Conflict of Interest Statement: The authors declare that the research was conducted in the absence of any commercial or financial relationships that could be construed as a potential conflict of interest.

Copyright $\odot 2018$ Critchley, Isalan and Mielcarek. This is an open-access article distributed under the terms of the Creative Commons Attribution License (CC BY). The use, distribution or reproduction in other forums is permitted, provided the original author(s) and the copyright owner are credited and that the original publication in this journal is cited, in accordance with accepted academic practice. No use, distribution or reproduction is permitted which does not comply with these terms. 